\title{
Quantitative Estimation of Adsorbed Water Layer on Austenitic Stainless Steel
}

\author{
Zaid Ali Subhi ${ }^{1)}$, Kanao Fukuda ${ }^{1,23)^{*}}$, Takehiro Morita ${ }^{3,4)}$ and Joichi Sugimura ${ }^{2,3,4)}$ \\ ${ }^{1)}$ Mechanical and Precision Engineering Department, Malaysia-Japan International Institute of Technology, \\ Universiti Teknologi Malaysia \\ Jalan Sultan Yahya Petra, Kuala Lumpur 54100, Malaysia \\ ${ }^{2)}$ International Research Center for Hydrogen Energy, Kyushu University \\ 744 Motooka, Nishi-ku, Fukuoka 819-0395, Japan \\ ${ }^{3)}$ International Institute for Carbon-Neutral Energy Research, Kyushu University \\ 744 Motooka, Nishi-ku, Fukuoka 819-0395, Japan \\ ${ }^{4)}$ Faculty of Mechanical Engineering, Kyushu University \\ 744 Motooka, Nishi-ku, Fukuoka 819-0395, Japan \\ *Corresponding author: fukuda.kl@utm.my
}

( Manuscript received 5 June 2015; accepted 17 August 2015; published 31 October 2015 )

\begin{abstract}
A study was carried out to estimate the thickness of the adsorbed water layer on the surface of austenitic stainless steel (JIS SUS316) in different atmospheric humidity conditions. The purpose of the study is to have a quantitative data on water adsorption that has influences on the tribological phenomena. The mass of stainless steel plate was determined by the weighting method as a function of the relative humidity (RH) in ambient atmospheric air. An experimental device consisting of atmospheric humidity controller and high sensitivity analytical balance with affixed airtight chamber was used for the purpose of the study. The result of water adsorption progression on SUS316 surface has been discussed and schematically demonstrated. It is clarified that the thickness of adsorbed water is significantly large to influence the tribological properties as demonstrated in a schematic model of the interface state of pin-on-disk prior to contact in high RH.
\end{abstract}

Keywords: water adsorption, relative humidity, tribological properties, SUS316, weighing method

\section{Introduction}

In the real-world of engineering applications, all components which are involved in mechanical contact are subject to the atmospheric environment changes. The changes of atmospheric environment have a substantial impact on the tribological performance of materials, especially metallic materials because of their highly reactive surface tendency. Since the tribological phenomena originate at micro and nano scale region, the influence of interface is critical because the surface-to-volume ratio is significantly large and surface properties dominate the tribological performance of materials. The adsorption of water on the metallic surface due to atmospheric humidity is considered one of the major influencing factor on the tribological properties [1] such as friction and wear as shown in our previous results [2] in Fig. 1. According to Goto [3], low humidity interferes with the adsorption of oxygen, reduces the rate of oxidation and so permits a greater amount of intermetallic contact. At high humidity, the greater amount of water adsorbed becomes sufficient to augment

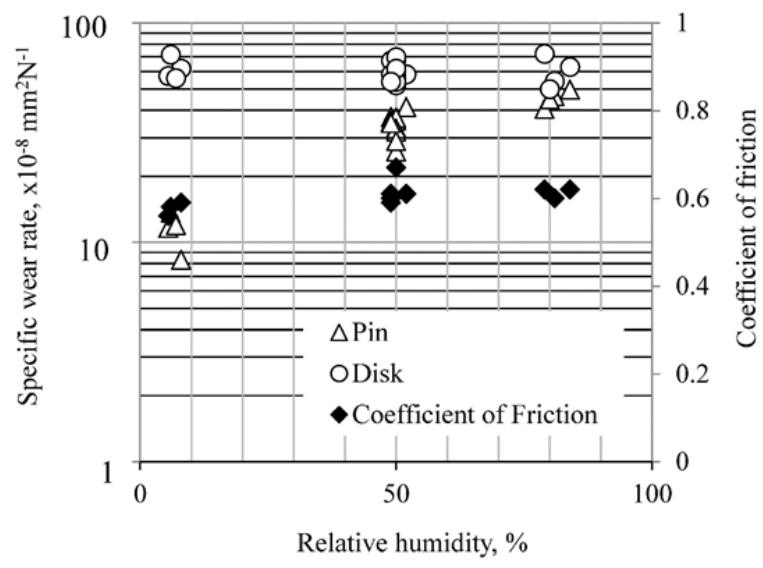

Fig. 1 The influences of relative humidity in the environmental air on the tribological properties of austenitic stainless steel [2] 


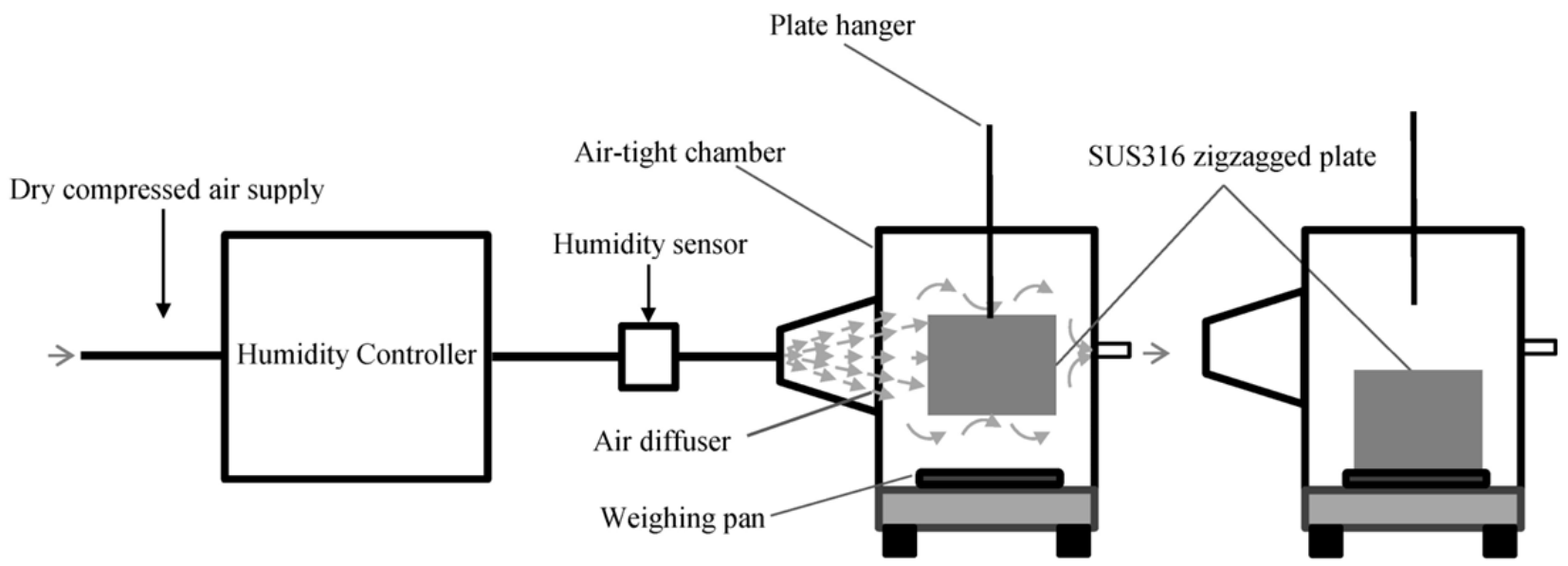

(a) Unloaded sample

(b) Loaded sample

Fig. 2 Schematic illustration of the measurement device

the protection against intermetallic contact. The presence of a thin liquid film with contact angle at the interface will result in so-called liquid-mediated adhesion [4]. The adhesion depends on the height of surface peaks and the thickness of adsorbed water [5]. It was found that friction force and wear in sliding can be influenced even by extremely low level as the several parts per million (ppm) concentration of the water [6-8]. Yet, in many articles about friction and wear experiments, the atmospheric humidity of the environment is rarely controlled [9]. The authors have found in the previous study significant influences of the atmospheric humidity on the initial stage of sliding [10]. It is suggested that adsorbed water has governed the state of contact in the very early stage of sliding which would determine the tribological performance in the later stages of sliding. In order to understand the phenomena precisely, a realistic estimation of adsorbed water thickness on the surface is necessary to be established. The structure of adsorbed water molecules has been studied extensively in ultrahigh vacuum conditions [11,12]. The knowledge obtained from these studies is useful in understanding water chemistry in the upper atmosphere or in space. However, it cannot be extrapolated to explain the phenomena occurring in normal ambient conditions. The suitable method to detect the changes of the adsorbed water layer in ambient is by weighing a metallic sample in different atmospheric environment and the changes of mass will be interpreted as the changes in the thickness of adsorbed water $[13,14]$. In this study, the thickness of the adsorbed water layer on SUS316 plate is quantitatively estimated by the weighing method. The results of this study have provided essential information to enhance the analysis on adhesion phenomenon and adhesive wear.

\section{Experimental Method}

The mass variations of adsorbed water layer on an austenitic stainless steel plate with different atmospheric humidity were estimated by the weighing method. The measurement device consists of air humidity controller and an airtight chamber affixed to an analytical balance (Max measurable mass of $420 \mathrm{~g}$ and resolution of $1 \mathrm{mg}$ ), as shown schematically in Fig. 2. The air humidity controller is capable to control the $\mathrm{RH}$ rate at the value between 4 and 95\% flexibly. The flow rate of humidity controlled air which is delivered to the testing chamber can be regulated from 1 to 8 liter per minute and the air flow rate in the experiments was adjusted to 5 liter per minute. The plate to be weighed is made of JIS SUS316 with dimensions of $300 \mathrm{~mm}$ length, $100 \mathrm{~mm}$ width and $0.5 \mathrm{~mm}$ thickness to guarantee a detectable mass of adsorbed water due to the limitation of the analytical balance minimum reading. The average surface roughness of the plate was measured with stylus type roughness tester. The radius of the stylus tip was $2 \mu \mathrm{m}$ and the angle was $6^{\circ}$. The measured average surface roughness of the plate was $115 \mathrm{~nm}$ Ra. The plate was tested as manufacturer surface machined finish but ultrasonically cleaned for $600 \mathrm{~s}$ in $50: 50$ mixtures of hexane and acetone. Later, the plate was dried in air of $\mathrm{RH} 30 \%$ for $1,800 \mathrm{~s}$. The plate was formed as a zigzag shape to maximize the surface area in the limited space of the chamber. The authors have confirmed experimentally that the sample can reach the saturation level of water adsorption at each RH \% before $1800 \mathrm{~s}$. Because the variation of adsorbed water to variation of environmental condition is relatively fast, in minutes time, if the surface is not porous [13]. Longer period of exposure to $\mathrm{RH} \%$ will not change the weight of the sample. Thus, the plate was exposed to each $\mathrm{RH}$ rate for 

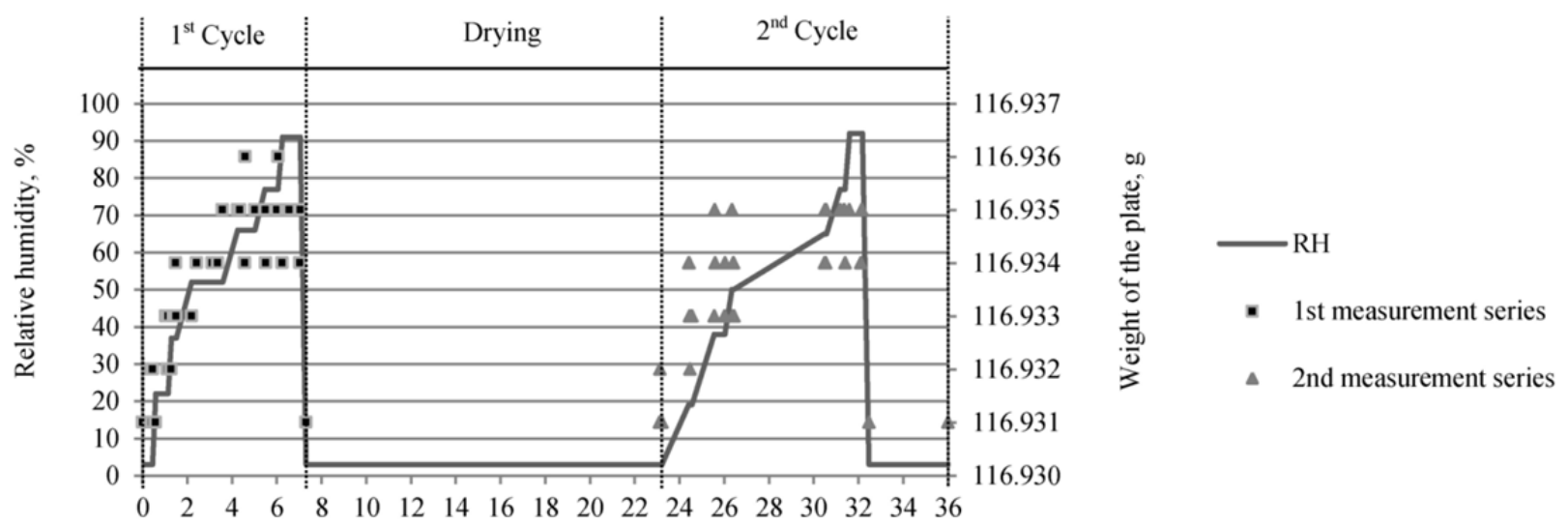

Time, h

Fig. 3 Changes in weight of SUS316 plate in different relative humidity as a function of time

a period of 1,800 s before the weight measurement was taken. For the weight measurement procedure, the plate was lifted from the weighing pan by the hangar to unload the balance and to let it exposed to the airflow with each experimental rate of $\mathrm{RH} \%$ as demonstrated in Fig. 2(a). After the necessary period, which is more than $1,800 \mathrm{~s}$, of the adsorbed water to get stabilized, the balance was calibrated and reading was set to zero and then the plate was released from the hanger and placed onto the weighing pan of the balance and the weight measurement was taken as shown in Fig. 2(b). After preparation of the measuring device (reaching a stable state of each $\mathrm{RH}$ rate, placing the plate onto the weighting pan), the mass of the plate was determined. The weight measurement of each $\mathrm{RH}$ rate was taken for 5 times to ensure the repeatability of the data. For each series of measurement cycle, the $\mathrm{RH}$ rate was adjusted one after the other gradually from low to high, and finally the $\mathrm{RH}$ rate which was adjusted first is reset for the new cycle of measurement. The cycle was repeated for twice to confirm the repeatability of the experiment cycle and tendency of water adsorption. Each measuring cycle took about 6 hours.

The real surface area of the plate is supposed to be relatively larger than the apparent surface area due to its roughness. The adsorption of water should be evaluated by the real surface area. The real surface area of the plate is evaluated based on the measurement data of the surface roughness. The real surface area is the result of multiplying the real length and real width. The real length and real width are the summation of the hypotenuses which is calculated by applying the Pythagorean theorem on the fluctuating line of the surface roughness. The calculated real surface area of the plate surfaces is around 1.09 times greater than the apparent surface area.

\section{Results and discussion}

The results of the plate weight changes as a function of $\mathrm{RH}$ rate of 2 cycles are shown in Figs. 3 and 4 . The minimum weight of the plate was recorded with $\mathrm{RH} 3 \%$ environment and the weight increased when $\mathrm{RH} \%$ sequentially increased up to $92 \%$. The changes of weight and $\mathrm{RH}$ as a function of time to understand the repeatability of the measurement cycle have been demonstrated in Fig. 3. The results show small deviations in each measurement that might be due to the power dissipated by electronic circuits of the balance or wind draft at the site of the balance. These deviations are presented as error bars in Fig. 4. The buoyancy effect on the weight measurement has been taken into consideration due to the density difference of the atmospheric air at different $\mathrm{RH} \%$. However, the buoyancy error in the current study has been already compensated in the calibration mass inside the analytical balance which was made of stainless steel, the same material of measurement plate. The atmospheric pressure was constant during the time of measurement as according to the report of Japan Meteorological Agency [15]; thus, the atmospheric pressure had no considerable influence on the buoyancy effect.

In Fig. 3, it is noticed that the weight of the plate has returned to the same original value before the second cycle of measurement. This behavior shows that the additional layer of adsorbed water on the surface might have been removed after the sample left again in $\mathrm{RH} 3 \%$. Therefore, the process was reversible and the adsorption was a physisorption; in addition, the chemisorption exists, but might be limited to a monolayer and not detectable by the analytical balance. According to the results shown in Fig. 3, the process of water adsorption was progressing rapidly when the $\mathrm{RH}$ was gradually increased from $\mathrm{RH} 3 \%$ to $70 \%$. After $\mathrm{RH} 70 \%$ the process of adsorption slows down, this is indicating that 


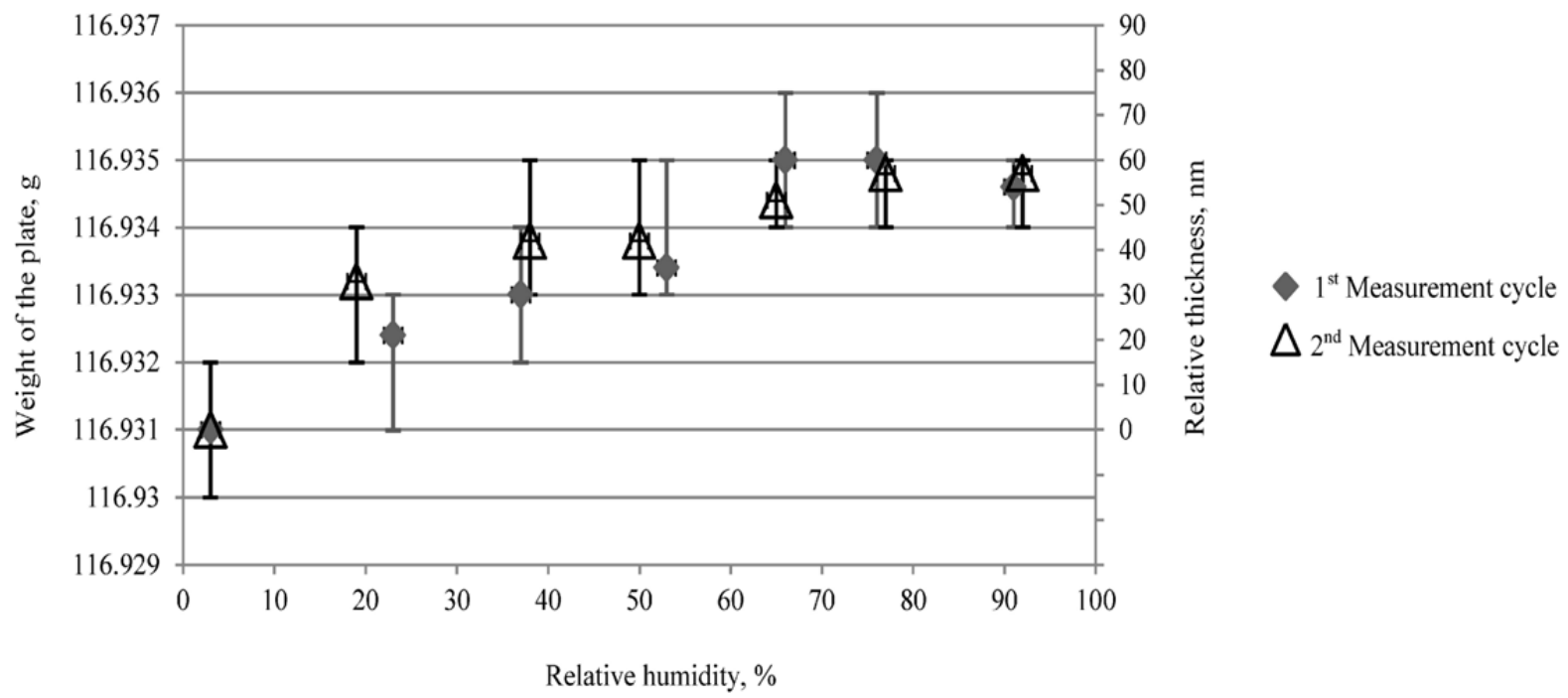

Fig. 4 Adsorption progress of water on the SUS316 surface as a function of relative humidity
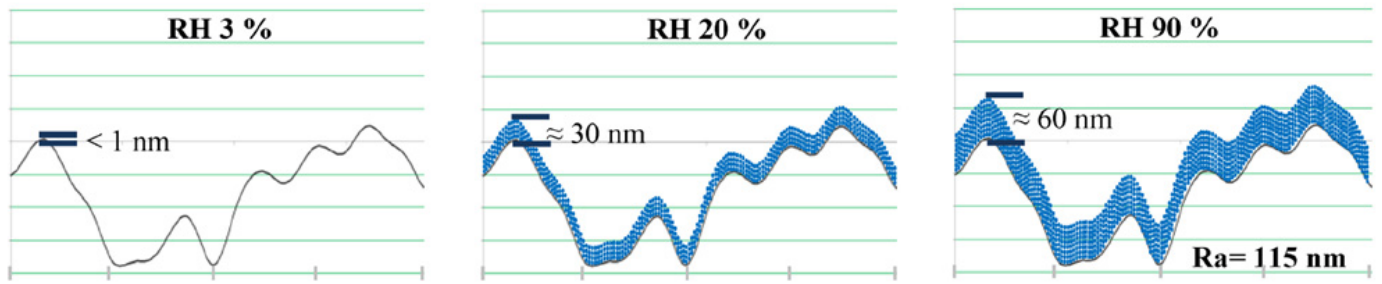

$100 \mathrm{~nm}$

$10,000 \mathrm{~nm}$

Fig. 5 Schematic illustrating the evolution of the adsorbed layer thickness with RH \% increases

the adsorption of water is reaching the saturation state.

As for the preliminary estimation of the adsorbed water thickness onto the plate, we assumed that the plate has no adsorbed water in RH 3\% (dry condition). The assumption was made due to the difficulty of attaining a completely dried sample in the chamber; however, it might be reasonable assuming that a few ice-like layers adsorb on the surface in low RH [16]. The relative thicknesses of adsorbed water as a function of RH were calculated using Eq. 1, the results are presented in Fig. 4.

$$
M=D * A * t
$$

Where $M$ represents the mass of adsorbed water on both sides of plate, $D$ denotes the density of adsorbed water, $A$ is the real surface area of the plate (1.09 times of apparent surface area) and $t$ is the thickness of the adsorbed water layer. The density of the water adsorbed on the surface was assumed to be equivalent to the density of the liquid water [16]. A schematic model which shows the evolution of water adsorption process on the surface of SUS316 in different RH environment is sketched in Fig. 5. It should be noted that the magnification of horizontal axis was much larger than that of the vertical axis in order to visualize the shape of the asperities with the adsorbed water. The model shows that the thickness of the adsorbed water layer is significantly big in high humidity environment compared to the profile of surface roughness. It is also important to highlight that the radius of the stylus that was used for the measurement of the surface roughness is bigger than the size of the subnanometer surface roughness. Thus, the subnanometer roughness was not considered in the calculation of the real surface area of the plate. However, we confirmed that the subnanometer surface roughness is much smaller than the thickness of adsorbed water and they will be completely covered with the thick layer of adsorbed water. Generally, the thickness of monolayer of water is around $0.3 \mathrm{~nm}$. As a number of layers description by dividing the total measured thickness over $0.3 \mathrm{~nm}$, in RH 20\% the plate was covered with approximately 100 layers of adsorbed water, while in RH $90 \%$ the plate is covered with approximately 200 layers of adsorbed 


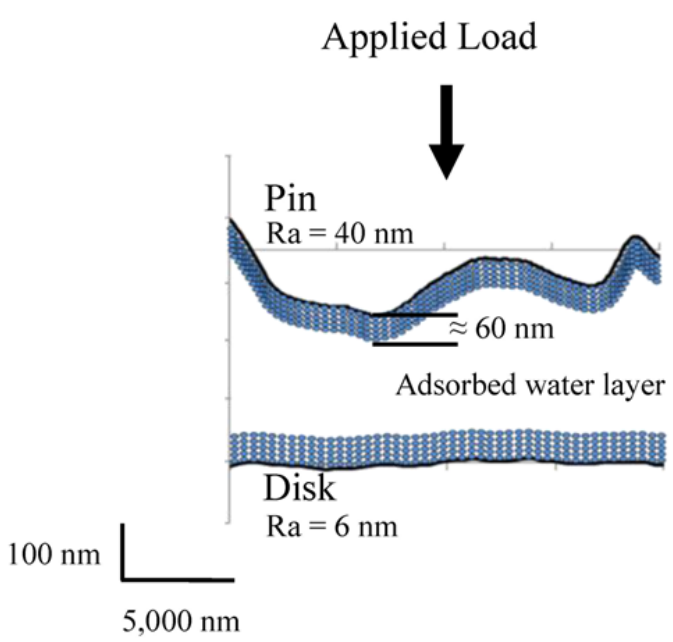

Pin-on-disk before contact

RH 90\%

Fig. 6 Schematic model of pin on disk interface prior to contact in high atmospheric humidity environment

water which is considerably thick. In our previous studies, the RH changes have had significant influences on the tribological properties in the very early and late stages of self-mated dry sliding of some metallic materials $[1,2,10]$. According to the results of the current study, the thickness of the adsorbed water layer is significantly big in high humidity environment as shown schematically in Fig. 6 which shows the realistic interface state of pin on disk in high RH in our previous study [10]. The authors have suggested that the thick adsorbed layer acted as a lubricating film on the very early stage of sliding to prevent direct metal to metal contact of asperities. In late stage of sliding, the thick layer of adsorbed water possibly enhanced the discharge process of adhered stuff as wear debris. Thus the wear rate was significantly increased in high humidity environment as shown earlier in Fig. 1.

\section{Conclusions}

In this study, the thickness of the adsorbed water layer on SUS316 has been quantitatively estimated in different condition of atmospheric humidity by using the weighing method. The results have provided necessary information for the interpretation of the influences of the adsorbed water on the state of the contact. The results showed that water adsorbed rapidly in the range of humidity from RH $20-70 \%$. Water adsorption from RH 70-90\% were relatively slow as the process, perhaps reached the saturation level. A realistic schematic model of the evolution of water adsorption process on the surface of SUS316 in different RH environment has been sketched based on the results of this study and real measurement of surface roughness.

\section{Acknowledgements}

This work is financially supported by JSPS KAKENHI Grant Number 26420080 (Japan) and Fundamental Research Grant Scheme (FRGS) No. PY /2013/01222 (Malaysia).

\section{References}

[1] Fukuda, K., Norose, S. and Sasada, T., "Effect of Humidity on Sliding Wear in Fe and Cu Rubbing System," Proceeding of the Twenty Eight Japan Congresses on Material Research, 1985, 89-92.

[2] Fukuda, K., Subhi, Z. A. and Morita, T., "Analytical Study on the Growth and Transfer of Adhesive Substances Generated on the Surface in the Early Stage of Sliding,” Wear, 330-331, 2015, 64-69.

[3] Goto, H. and Buckley, D. H., "The Influence of Water Vapour in Air on the Friction Behaviour of Pure Metals During Fretting," Tribology International, 18, 4, 1985, 237-245.

[4] Bhushan, B., "Adhesion and Stiction: Mechanisms, Measurement Techniques, and Methods for Reduction,” Journal of Vacuum Science \& Technology B, 21, 6, 2003, 2262-2296.

[5] Bowden, F. P. and Tabor, D., "The Friction and Lubrication of Solids," Oxford University Press, 2009, 304.

[6] Fukuda, K., Hashimoto, M. and Sugimura, J., "Effects of Trace Impurification in Hydrogen Environment on Tribological Properties of Steels," Journal of Japanese Society of Tribologists, 55, 1, 2010, 53-61 (in Japanese).

[7] Fukuda, K., Hashimoto, M. and Sugimura, J., "Friction and Wear of Ferrous Materials in a Hydrogen Gas Environment,” Tribology Online, 6, 2, 2011, 142-147.

[8] Fukuda, K. and Sugimura, J., "Influences of Trace Water in a Hydrogen Environment on the Tribological Properties of Pure Iron,” Tribology Online, 8, 1, 2013, 22-27.

[9] Lancaster, J. K., "A Review of the Influence of Environmental Humidity and Water on Friction, Lubrication and Wear,” Tribology International, 23, 6, 1990, 371-389.

[10] Subhi, Z. A., Morita, T. and Fukuda, K., "Analysis of Humidity Effect on Early Stage of Sliding," Procedia Engineering, 68, 2013, 199-204.

[11] Stevenson, K. P., Kimmel, G. A., Dohnalek, Z., Smith, R. S. and Kay, B. D., "Controlling the Morphology of Amorphous Solid Water," Science, 283, 5407, 1999, 1505-1507.

[12] Henderson, M.A., "The Interaction of Water with Solid Surfaces: Fundamental Aspects Revisited," Surface Science Reports, 46, 1-8, 2002, 1-308. 
[13] Kochsiek, M., "Measurement of Water Adsorption Layers on Metal Surfaces,” Metrologia, 18, 3, 1982, 153-159.

[14] Picard, A. and Fang, H., "Methods to Determine Water Vapour Sorption on Mass Standards," Metrologia, 41, 4, 2004, 333-339.
[15] http://www.data.jma.go.jp/fcd/yoho/data/hibiten/2 014/1407.pdf (in Japanese)

[16] Asay, D. B. and Kim, S. H., "Evolution of the Adsorbed Water Layer Structure on Silicon Oxide at Room Temperature," The Journal of Physical Chemistry B, 109, 35, 2005, 16760-16763. 